\title{
Impactos de uma taxação ambiental sobre a demanda no transporte aéreo brasileiro
}

\author{
Carolina Barbosa Resende ${ }^{1}$, Alessandro Vinícius Marques de Oliveira ${ }^{2}$ \\ ${ }^{1}$ Instituto Tecnológico de Aeronáutica - ITA, São José dos Campos, SP, Brasil, carolina.barres@gmail.com \\ 2Instituto Tecnológico de Aeronáutica - ITA, São José dos Campos, SP, Brasil, alessandro@ita.br
}

\section{Recebido:}

26 de outubro de 2016

Aceito para publicação:

23 de março de 2017

Publicado:

31 de agosto de 2017

Editor de área:

Li Weigang, UnB

\section{Palavras-chaves:}

Econometria.

Demanda por transporte aéreo.

Emissões de aeronaves.

\section{Keywords:}

Econometrics.

Demand for air transport.

Emissions of aircraft

DOI:10.14295/transportes.v25i2.1271

\begin{abstract}
RESUMO
Nos últimos anos, o tema das emissões de gases do efeito estufa e seus efeitos potencialmente danosos sobre o planeta entrou definitivamente na pauta de governos ao redor do mundo, com destaque aos encontros internacionais realizados que visaram acordar entre os países formas de equacionar o problema. Em se tratando de aeronaves, sabese que a queima do combustivel as tornam fontes emissoras importantes de tais gases. Sabe-se que a taxação das passagens aéreas, por afetar diretamente a geração de viagens aéreas, tem potencial de atuar como instrumento arrefecedor de demanda e, por decorrência, do número de viagens e voos e, em última instância, do consumo de combustivel. $O$ presente trabalho visa quantificar essa relação, simulando a inserção de um imposto ambiental sobre o preço do querosene de aviação e seu impacto indireto sobre a demanda por transporte aéreo. Estimou-se, utilizando modelagem econométrica, que a demanda cairia frente a um aumento nos preços decorrente do imposto, mas que a reação ocorre de forma assimétrica conforme o mercado. Observou-se que rotas com perfil mais turísticos sofreriam uma redução de 6,06\% para uma taxa de 10EUR, enquanto uma rota com perfil mais corporativo sofreria queda de $2,35 \%$ para mesma taxa.
\end{abstract}

\section{ABSTRACT}

In recent years, the greenhouse gases emissions issue and its potentially harmful effects on the planet have definitively entered the government agenda around the world, especially to international meetings that aimed an agreement to solve the problem between countries. In the airline industry, it is known that the fuel burn is an important source of emission for those gases. It is known that the airline tickets pricing taxation, by directly affecting the air travel generation, has the potential to act as a demand diminisher instrument and, consequently, the number of trips and flights and, ultimately, fuel consumption. The present paper aims to quantify this relationship, simulating the inclusion of an environmental tax on aviation kerosene price and its indirect impact on air transport demand. It was estimated, using an econometric model, that the demand would fall with a price increase due to the tax, but this reaction happens in an asymmetric way according to the market characteristics. It was noticed that routes with a more tourist profile would notice a $6.06 \%$ fall in the demand to a 10EUR tax, while a more business route would decrease $2.35 \%$ for the same 10 EUR tax.

\section{INTRODUÇÃO}

Protocolo de Kyoto, Greenhouse Gas, COP21 e COP22 são exemplos de tratados internacionais que abordaram o tema emissões de gases poluentes devido às preocupações com efeito estufa e aquecimento global. Em 2015 a 21ํㅡ Conferência das Partes (COP21) da Convenção-Quadro das Nações Unidas sobre Mudança do Clima foi realizada e abordou o tema das emissões internacionalmente, incluindo o transporte aéreo nessa preocupação, em 2016 a 22를 Conferência das Partes (COP22) continuou com a preocupação climática e deu continuidade à implementação do acordo da COP21.

Silva e Paula (2009) disseram que dentre os gases responsáveis pelo efeito estufa tem-se dióxido de carbono $\left(\mathrm{CO}_{2}\right)$, metano $\left(\mathrm{CH}_{4}\right)$, óxido nitroso $\left(\mathrm{N}_{2} \mathrm{O}\right)$, vapor de água, clorofluorcarbono (CFC) e ozônio $\left(\mathrm{O}_{3}\right)$. 
As emissões da aviação são compostas por cerca de $71 \%$ de $\mathrm{CO}_{2}, 28 \%$ de $\mathrm{H}_{2} \mathrm{O}$ e menos de $1 \%$ por todos os outros gases (Schumann, 2002).

Segundo Pavão (1997) as principais fontes emissoras de $\mathrm{CO}_{2}$ são a queima de combustível fóssil e a queima de biomassa. Sendo assim, a aviação é uma fonte emissora devido à queima de combustível durante os processos de taxiamento das aeronaves e durante o percurso do voo. Simões e Schaeffer (2005) sugerem que aproximadamente 3,5\% das emissões mundiais de gás carbônico são provenientes da aviação.

Com o intuito de reduzir as emissões, um dos mecanismos de mitigação possíveis seria a cobrança de um imposto sobre o consumo de combustível de aviação sob as companhias aéreas. Essa medida vem sendo estudada por governos de alguns países da União Europeia em uma aplicação conhecida como taxação ambiental (Eurostat, 2013). Sob a ótica empresarial, a taxação ambiental acaba representando um custo adicional para as operadoras, tornando-as menos competitivas. Tem-se, entretanto, que conforme o comportamento das empresas, segundo Hofer et al. (2010), a taxação pode em sequência impactar os preços, a demanda e, em última instância, a lucratividade do setor, uma vez que as empresas poderão arcar com as taxas ou efetuar repasses integrais, parciais ou mesmo mais que proporcionais ao consumidor, dependendo da dinâmica de mercado.

O objetivo deste trabalho é estudar o impacto gerado na demanda devido a uma possível taxação ambiental sobre as emissões de gases gerados pelo tráfego aéreo brasileiro. Para isso um modelo econométrico foi desenvolvido e analisado, levando à conclusão que a aplicação da taxação ambiental gera um aumento nos custos, aumentando consequentemente o preço, e isso leva a uma queda da demanda, porém a reação varia com diferentes características do mercado, ou seja, destinos mais turísticos perdem proporcionalmente mais passageiros do que destinos corporativos.

0 artigo é estruturado da seguinte forma: a segunda seção apresenta a revisão da literatura e o modelo conceitual. 0 desenvolvimento do modelo empírico é trazido na Seção 3. A Seção 4 apresenta os resultados do modelo. A checagem da robustez e as limitações do trabalho são trazidos na Seção 5. E depois dessas seções as conclusões do artigo são apresentadas.

\section{ESTRUTURA TEÓRICA}

As perguntas de pesquisa são uma forma de apresentar o objetivo do artigo, o qual é entender a relação entre taxação ambiental e demanda do transporte aéreo. Sendo assim, procura-se responder a seguinte pergunta de pesquisa: a aplicação de uma taxação ambiental gera reações assimétricas na demanda? Em outras palavras, ao analisar diferentes mercados, a demanda reagirá de formas diferentes entre eles ou será uma reação regular?

É esperado que as tarifas aéreas aumentem com a implementação de uma taxação ambiental e que a demanda decresça com isso, mas também é esperado uma reação irregular entre diferentes mercados. Isso porque existem diferentes perfis de passageiros para cada rota, mas também existem fontes de assimetrias diversas como a presença de concorrência intermodal ou a presença de um concorrente low cost que podem afetar a eficácia relativa da implementação da taxação ambiental.

\subsection{Revisão da Literatura}

O efeito estufa é um fenômeno ocasionado pela emissão de gases. 0 aumento das emissões tende a intensificar o efeito causado, impactando também a vida humana devido ao aquecimento terrestre e às doenças predispostas por ela. Gases de emissão antropológica como gás carbônico podem ter suas emissões controladas, reduzindo e evitando assim diversos problemas, dentre eles reduzindo o efeito estufa, o qual veio aumentando ao longo dos últimos anos (Silva e Paula, 2009).

A sociedade busca a redução das emissões de gases por dois motivos, (i) efeito estufa (Hofer et al. 2010) e (ii) saúde humana (Williams, 2002). Segundo Simões e Schaeffer (2005), cerca de 13\% das emissões antropogênicas são provenientes do setor de transporte, enquanto a aviação é responsável por 
cerca de 3,5\% de todo o dióxido de carbono emitido. Betiolo et al. (2009) argumentaram que, apesar da aviação contribuir com pequenos índices de emissões, os gases poluentes lançados por aeronaves são potencializados por serem emitidos em grande altitude, o que intensifica a concentração de gases na atmosfera.

A medida que a demanda por viagens aumenta, um maior número de voos pode ser ocasionado, e com isso o consumo de combustível é intensificado, aumentando a queima provocada e consequentemente as emissões de poluentes gerado. Outros aspectos que influenciam nas emissões dos voos, porém não limitados a estes são: distância da rota, modelo de aeronave e empresa aérea (Grampella et al.,2016).

A taxação ambiental é uma taxa ou imposto aplicada visando melhorias ambientais. Segundo Bosquet (2000), a taxação ambiental é voltada para algo que é indesejável, é aplicada para diminuir o desperdício, a poluição e a emissão de gases, e pode atingir melhorias tanto ambientais quanto econômicas.

O estudo de Brouwer et al. (2008) e Jou e Chen (2015) mostram que parte dos passageiros se sensibilizam com o assunto de emissões e poluição e por esse motivo, quando aplicado taxas ambientais, eles estão dispostos a pagar mais pelo produto. Essa reação dos clientes também é de suma importância, pois com isso pode-se concluir que a procura pelo produto não cai de forma linear com o aumento do preço, e a reação dos consumidores é influenciada pelo motivo do aumento nos preços das passagens. Além disso, devido às taxações, existe uma variação do valor que os passageiros estão dispostos a pagar a mais em diferentes rotas.

Segundo Andersen (2010) alguns países membros da União Europeia (UE) aplicam taxas por tonelada de $\mathrm{CO}_{2}$ emitido. 0 governo Irlandês, segundo o mesmo autor, aplicava uma taxa de 15 euros (EUR), enquanto a França estava analisando uma taxa de 17EUR já em 2010. Iannone (2011) em seu estudo analisou taxações entre 10 e 30EUR devido às variações existentes entre países da UE.

0 principal motivo para se taxar é o de reduzir as emissões e a concentração desses gases na atmosfera. Porém, a taxação causa diversas reações no mercado, por isso deve ser bem analisada antes de sua aplicação. Entre as reações da taxação tem-se a alteração nos custos das empresas e consequentemente as tarifas aplicadas podem alterar, o que pode causar uma queda da demanda pelo transporte aéreo.

Sabendo disso, a oferta e a demanda sofrem impactos devido à taxação ambiental, porém outras características do mercado também impactam no mesmo período, por exemplo se em período de crise financeira for aplicada uma taxação ambiental, então as reações esperadas serão diferentes se comparadas com o mesmo período sem taxação.

\subsection{Modelo Conceitual}

A taxação ambiental é aplicada visando reduzir efeitos ambientalmente indesejáveis (Bosquet, 2000). Essa taxação vem sendo aplicada e estudada em diversos mercados com a intenção de reduzir as emissões de gases causadores do efeito estufa. Apesar de existirem diversos gases que provocam tais emissões como $\mathrm{CO}_{2}$, metano e óxido nitroso, o foco desse estudo se dá unicamente nas emissões de $\mathrm{CO}_{2}$, gás de origem antropológica amplamente emitido.

É perceptível que a taxação ambiental além de impactos ambientais (redução das emissões de gases), geram tanto impactos econômicos (aumento do custo da empresa) quanto sociais (aumento dos preços das passagens aéreas no caso da transmissão do custo para o consumidor final). Este é mais um dos motivos para a taxação ser avaliada com cuidado antes de ser aplicada.

Elasticidade-preço da demanda é um conceito vindo da economia que mede a variação percentual da quantidade demandada de um produto devido à variação percentual no preço. Essa elasticidade pode ser útil para informar o comportamento da demanda segundo um choque no preço causado pela taxação ambiental. Ou seja, para um aumento nos custos, caso esse valor seja repassado aos consumidores finais, é esperado que o mercado reaja de forma diferente conforme características específicas.

A taxação ambiental pode causar reações diversas na demanda e na oferta devido ao fato da mesma se tornar um custo a mais para as empresas aéreas. Com isso, a empresa se vê frente a duas claras alter- 
nativas: (i) transmissão dos novos custos aos passageiros, ou (ii) arcar com o aumento dos custos. Na primeira opção a empresa aérea aumenta as tarifas cobradas no trecho voado; e na segunda opção ocorre a queda do lucro. As empresas normalmente se comportam de uma mesma maneira, independentemente do mercado em que atuam, pois tal comportamento visa o lucro. Portanto, é considerado que a redução dos lucros é algo que as empresas não aceitam facilmente, logo neste estudo considera-se que os novos custos são repassados para os passageiros.

Um dos motivos para o estudo sobre os impactos dessa taxação se dá ao fato do impacto não ser apenas sob a aviação. Existe a possibilidade de a taxação aumentar as tarifas a ponto de a demanda pelo transporte aéreo cair, e isso pode levar a uma consequente queda do turismo em determinadas regiões, queda de negócios, aumento do tráfego de carros e ônibus, dentre outros. Nesses casos o impacto perante as emissões pode não ser relevante diante de todos os outros impactos causados. Segundo Hofer et al. (2010) a troca no modal de transporte pode até mesmo fazer com que as emissões de gases aumentem, ou seja, as emissões por aeronaves podem diminuir, porém o aumento da procura pelo modal rodoviário pode gerar um aumento nas emissões que supere a economia gerada pelas aeronaves. Caso isso aconteça, a taxação estaria gerando um efeito contrário ao proposto desejado.

É esperado que diferentes tipos de passageiros se comportem de maneira diferente ao aumento nas tarifas, baseado nisso algumas hipóteses são levantadas. A análise e o teste formal dessas hipóteses foram realizados por meio de uma modelagem econométrica e por simulações no mercado aéreo brasileiro.

- H1: A reação da demanda ao aumento da taxação não é linear: a medida que há um aumento na taxação existe uma maior redução de demanda, porém essa reação não se dá de forma constante e linear;

- H2: Perfil dos passageiros e reação da demanda: passageiros com diferentes motivos de viagens reagem de forma diferente.

- H3: Região destino do passageiro e reação da demanda: no Brasil algumas regiões são destinos principalmente de clientes a turismo e outros a negócios. Portanto, diferentes reações são esperadas para diferentes regiões destino da viagem;

- H4: Entrada de LCC e reação da demanda: empresas LCC são conhecidas por aplicarem tarifas mais baixas, portanto a entrada desse tipo de empresa no mercado faz com que a demanda reaja de forma diferente ao mesmo mercado sem a presença das LCC;

- H5: Instabilidade econômica e demanda: períodos de instabilidade financeira para o setor aéreo geram uma maior queda na demanda, o que pode estar relacionado ao receio dos clientes com o mercado.

\section{DESENVOLVIMENTO DO MODELO EMPÍRICO}

Sabendo que diversos fatores alteram as emissões e as escolhas de clientes (Grampella et al., 2016), foi possível realizar um estudo para ver os impactos gerados na demanda pelo transporte aéreo doméstico brasileiro de acordo com alterações de determinados fatores.

\subsection{Aplicação}

0 modelo proposto foi aplicado no mercado aéreo doméstico brasileiro. Para tal, uma análise econométrica de preço foi realizada, para saber como demanda reage a preço, e a partir disso simulações de taxações foram realizadas, para então ser analisada a reação da demanda às taxações ambientais.

\subsection{Dados}

A base de dados consiste em um painel de dados com variáveis que vieram de diversas fontes, incluindo Agência Nacional de Aviação Civil (ANAC), Instituto Brasileiro de Geografia e Estatística (IBGE), IPEA data, dentre outros, e os dados cobrem o período de janeiro de 2002 a dezembro de 2013. 


\subsection{Modelo Empírico}

O modelo empírico utilizado é apresentado na Equação 1. Na Equação 1 todas as variáveis foram analisadas para uma rota $k$ em um mês t. A rota $k$ é na verdade representada não por aeroportos, mas por par de cidades e varia de 1 a 225; e t, sendo o tempo, varia de 1 a 144, ou seja, a base possui dados de 225 pares de cidades domésticas e de 144 meses (12 anos). E $\beta$ é a elasticidade de cada variável, sendo até então parâmetros desconhecidos. Na base, todos os dados de preço e custos já estão em reais (BRL) trazidos a valor presente de janeiro de 2015 utilizando deflator IPCA do IBGE.

$$
\begin{aligned}
& \ln r p k_{k t}=\beta_{0}+\beta_{1} \ln \text { yield }_{k t}+\beta_{2} \ln \text { grav income }_{k t}+\beta_{3} \ln \text { grav pop density }{ }_{k t}
\end{aligned}
$$

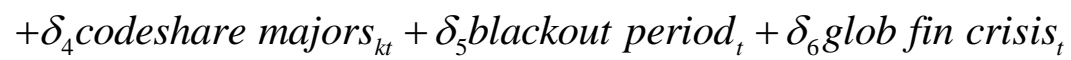

$$
\begin{aligned}
& +\delta_{7} \text { LCC }_{\text {Startup }}+\beta_{8} \ln \text { yield }_{k t} x \text { intermod }_{k}+\beta_{9} \ln \text { yield }_{k t} x \text { business }_{k} \\
& +\beta_{10} \ln \text { yield }_{k t} x \text { LCCstartup }_{k t}+\text { saz }_{\text {om }}+s a z_{d m}+\varepsilon_{k t}
\end{aligned}
$$

$r p k_{\mathrm{kt}}$ corresponde ao número de passageiros-quilômetros transportados no par de cidades $k$ e no mês $t$. Fonte: ANAC (2002-2013).

$y i e l d_{k t}$ é o valor médio pago por passageiro por quilômetro em um par de cidades $k$ e no mês $t$. A partir de julho de 2009 foi usado a receita média por passageiro-quilômetro por aeronave nas rotas. Fonte: ANAC (2002-2013).

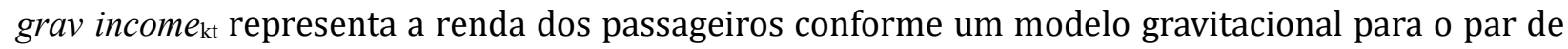
cidades $k$ e no mês $t$. Valores interpolados para obter uma periodicidade mensal. Fonte: IBGE.

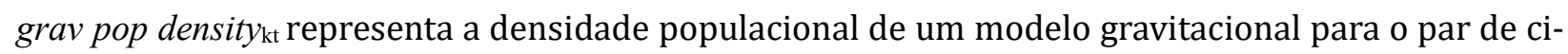
dades $k$ e no mês $t$. Valores interpolados para obter uma periodicidade mensal. Fonte: IBGE.

codeshare majorskt representa a existência de empresas operando codeshare no par de cidades $k$ no mês $t$, mais especificamente trata das rotas e períodos em que Tam e Varig operaram em regime codeshare entre março de 2003 e abril de 2005. Fonte: Secretaria de Acompanhamento Econômico do Ministério da Fazenda (SEAE/MF).

blackout period $d_{t}$ representa uma variável dummy que indica se o mês $t$ em análise passa pelo período do chamado "apagão aéreo" brasileiro. Esse período $t$ representa um valor unitário da dummy para o intervalo entre outubro de 2006 até julho de 2007. Fonte: Oliveira et al. (2011).

glob fin crisis té uma variável dummy que indica a crise econômica global - a chamada "crise do subprime" norte-americana e que se alastrou por todo o mundo - no mês $t$. Para fins de configuração do modelo empírico, considerou-se os impactos dessa crise no último trimestre de 2008, quando o total de passageiros transportados pagos pelo transporte aéreo no Brasil encerrou uma sequência de vinte semestres de crescimento ininterrupto, tendo retraído $2,1 \%$ em relação ao mesmo período de 2007. Já no primeiro semestre de 2009, houve uma retomada do crescimento, com variação positiva de $2 \%$ em relação ao primeiro trimestre de 2008. Fonte: ANAC (2002-2013), com cálculos próprios dos autores.

LCCstartup $_{\mathrm{kt}}$ variável dummy que indica a presença de empresas do tipo low cost, mais especificamente presença da empresa Azul para a rota representada pelo par de cidades $k$ no mês $t$. Fonte: ANAC (2002-2013).

intermod $_{\mathrm{k}}$ indica a proporção de passageiros que já fizeram a rota com diferentes modais de transporte. Fonte: FIPE, 2009.

business $\mathrm{k}_{\mathrm{k}}$ representa a proporção de passageiros voando a negócios no voo analisado. Fonte: FIPE, 2009.

$s a z_{\mathrm{om}}$ e $s a z \mathrm{dm}$, são respectivamente sazonalidade regional na origem o no mês $m$ e sazonalidade regional no destino $d$ e no mês $m$, e são tratados no modelo como efeito fixo.

$\varepsilon_{\mathrm{kt}}$ é o termo de erro que inclui as variáveis não observáveis para o par de cidades $k$ e no mês $t$. 
A Tabela 1 apresenta as estatísticas descritivas das variáveis apresentadas na Equação 1.

Tabela 1: Estatística descritiva das variáveis contínuas

\begin{tabular}{cccccc}
\hline Variável & Unidade & Média & Desvio Padrão & Min & Max \\
\hline rpk & Passageiros*km & 605225,50 & 871604,00 & 3294,61 & 7971701,00 \\
yield & BRL & 58,58 & 30,88 & 13,28 & 299,61 \\
grav income & BRL & 484844,30 & 868263,10 & 5553,74 & 11600000,00 \\
grav pop density & Habitantes/km² & 162338,70 & 251414,40 & 17,69 & 1738978,00 \\
codeshare majors & index [0,1] & 0,17 & 0,37 & 0,00 & 1,00 \\
blackout period & index [0,1] & 0,07 & 0,26 & 0,00 & 1,00 \\
glob fin crisis & index [0,1] & 0,03 & 0,17 & 0,00 & 1,00 \\
LCC startup & index [0,1] & 0,20 & 0,40 & 0,00 & 1,00 \\
intermod & $\%$ & 11,41 & 13,78 & 0,00 & 100,00 \\
Business & $\%$ & 55,40 & 17,29 & 0,00 & 100,00 \\
\hline
\end{tabular}

\subsection{Estratégia de Estimação}

No desenvolvimento da modelagem econométrica, foi realizada uma bateria de testes estatísticos de forma a adequar o modelo às especificidades do processo gerador dos dados. Em particular, foram realizados testes de hipótese quanto à presença de problemas de heteroscedasticidade e autocorrelação dos resíduos, típicos de bases estruturadas em forma de painel de dados. Adicionalmente, foram realizadas análises referentes à endogeneidade de regressores e as formas de endereçamento do problema.

\subsubsection{Heteroscedasticidade e Autocorrelação}

A heteroscedasticidade é uma, na qual a variância do regressando (e por consequência, do termo aleatório), condicional ao valor dos regressores, não é um valor constante, mas uma função dos próprios regressores (Wooldridge, 2013). Já o problema de autocorrelação ocorre quando os resíduos não são independentes temporalmente (ou espacialmente) em cada observação. Ambos os problemas configuram situações de quebra de hipótese do Modelo Clássico de Regressão Linear, com risco de falso positivo nos testes de hipótese referentes à significância estatística dos regressores, e devem ser endereçados de forma a obter uma estimativa consistente dos erros padrões dos estimadores. Com relação à heteroscedasticidade foram implementados os testes de White/Koenker e Breusch-Pagan/Godfrey/Cook-Weisberg e o modelo rejeitou a hipótese nula do teste, o que indica a presença de heteroscedasticidade. Com relação à autocorrelação dos resíduos, foi utilizado o teste Cumby-Huizinga, que indicou a presença de autocorrelação de ordem 3. Para o equacionamento dos problemas da regressão, utilizou-se o procedimento do estimador de variância consistente à heteroscedasticidade e autocorrelação de Newey-West (Wooldridge, 2013). Na configuração do equacionamento do problema da autocorrelação, utiliza-se a largura de banda (bandwidth), um parâmetro de suavização em uma matriz de pesos cujo objetivo é obter um estimador consistente da matriz de variância e covariância. Em geral, a escolha da banda é feita pelo modelador de acordo com sugestões da literatura, sendo que a definição de parâmetros altos envolve estabelecer defasagens mais distantes - o que pode aumentar a variância, mas, ao mesmo tempo, reduzir o viés do estimador - Wilhelm (2015). Greene (2007, p. 643) sugere o uso da banda igual ao tamanho da amostra elevado a 0.25 , procedimento adotado no presente trabalho.

\subsubsection{Endogeneidade e Variáveis Instrumentais}

A endogeneidade é um problema de modelagem econométrica em que se configura a correlação de uma variável regressora com o termo de erro $\varepsilon k t$. A presença de variáveis endógenas pode gerar resultados viesados dos coeficientes estimados, e para tratar essas ocorrências o modelo deve ser instrumentado. De acordo com a literatura, no modelo de demanda apresentado, as variáveis endógenas são preço e todas as suas interações. Através do método dos momentos generalizados em dois estágios (2SGMM) é possível tratar a endogeneidade com o emprego de variáveis instrumentais. 
O modelo foi estimado através do método 2SGMM ajustado para heteroscedasticidade e autocorrelação. Foram empregados também o procedimento Newey-West e efeitos fixos com controle de sazonalidade.

Os primeiros instrumentos utilizados ao considerar modelos de oferta e demanda foram custos, uma vez que esses impactam nos preços e na demanda, porém além deles, outros instrumentos foram buscados para uma melhor análise dos resultados. Nesse sentido instrumentos do tipo Hausman (apud Bresnahan e Gordon, 1996) foram utilizados. Esse tipo de instrumento assume que a variável a ser instrumentada está correlacionada entre os mercados, porém não está correlacionada ao erro. Hausman (apud Bresnahan e Gordon, 1996) relata, adaptado para a variável endógena desse modelo, que existem choques de preço entre as cidades, porém esses choques são independentes, com isso o que se diz é que os preços em diferentes períodos, para uma mesma rota, estão correlacionados.

\subsection{Transmissão}

A taxação ambiental como já dito anteriormente impacta diretamente nos custos da empresa, no entanto para os custos impactarem no preço uma forma de transmissão deve ser analisada. Com base nisso o poder de mercado foi estudado, o qual se trata da capacidade que uma empresa tem de escolher o preço de mercado do produto. Uma das medidas para esse poder de mercado é definido pelo índice de Lerner, segundo Motta (2004, p. 123) dado pela Equação 2.

$$
\mathrm{L}=\frac{\mathrm{p}-\mathrm{c}}{\mathrm{p}}=\frac{\mathrm{HHI}}{\varepsilon}
$$

Em que HHI: Herfindahl-Hirschmann Index

$p$ : preço de equilíbrio do mercado

$c$ : custo marginal médio de mercado assumindo homogeneidade

Com base na Equação 2 existe uma relação que foi baseada no índice Lerner, o qual é apresentado na Equação 3 devido à decorrência de um modelo de oligopólio.

$$
\mathrm{L}_{\mathrm{i}}=\frac{\mathrm{m}_{\mathrm{i}}}{\varepsilon}
$$

Em que $L_{i}:$ índice de Lerner de uma empresa i;

$m_{i}$ : Market share da empresa i;

$\varepsilon$ : elasticidade da demanda do mercado com relação ao preço.

O HHI é um índice que avalia o grau de concentração do mercado, permitindo avaliar não só uma empresa, mas toda a indústria.

Uma relação preço/custo, tratada por passthrough, é apresentada na Equação 4, e é com base nela que a taxação ambiental é transmitida para as tarifas médias das passagens aéreas.

$$
\frac{\mathrm{p}}{\mathrm{c}}=\frac{1}{1-\frac{\mathrm{HHI}}{\varepsilon}}
$$

\section{RESULTADOS}

Com base na Equação 1 a Tabela 2 foi gerada. A Tabela 2 mostra os resultados da regressão obtida através do estimador 2SGMM, estimador esse que trata a endogeneidade das variáveis necessárias. 
Tabela 2: Resultados (estimador 2SGMM) - modelo principal

\begin{tabular}{|c|c|}
\hline & (1)_Inrpk \\
\hline \multirow[t]{2}{*}{ In grav pop density } & $1,2925 * * *$ \\
\hline & {$[0,079]$} \\
\hline \multirow[t]{2}{*}{ In grav income } & $0,1947 * * *$ \\
\hline & {$[0,026]$} \\
\hline \multirow[t]{2}{*}{ In yield } & $-1,0036 * * *$ \\
\hline & {$[0,166]$} \\
\hline \multirow[t]{2}{*}{ codeshare majors } & $-0,0469 * * *$ \\
\hline & {$[0,014]$} \\
\hline \multirow[t]{2}{*}{ blackout period } & $-0,2425 * * *$ \\
\hline & {$[0,016]$} \\
\hline \multirow[t]{2}{*}{ global fin crisis } & $-0,0216$ \\
\hline & {$[0,015]$} \\
\hline \multirow[t]{2}{*}{ LCC startup } & $1,9179 * * *$ \\
\hline & {$[0,353]$} \\
\hline \multirow[t]{2}{*}{ In yield $\mathrm{x}$ intermod } & $-0,0236 * * *$ \\
\hline & {$[0,008]$} \\
\hline \multirow[t]{2}{*}{ In yield $x$ business } & $0,0094 * * *$ \\
\hline & {$[0,003]$} \\
\hline \multirow[t]{2}{*}{ In yield $\mathrm{x}$ LCC start } & $-0,5146 * * *$ \\
\hline & {$[0,097]$} \\
\hline R2 ajustado & 0,9267 \\
\hline Estatística RMSE & 0,3404 \\
\hline Estatística F & 1091,429 \\
\hline KP estatística & 137,6413 \\
\hline KP_P valor & 0,0000 \\
\hline J estatística & 0,3213 \\
\hline J_P valor & 0,8516 \\
\hline Estatística CD Weak & 41,8366 \\
\hline Estatística KP Weak & 24,3265 \\
\hline Número de observações & 25347 \\
\hline
\end{tabular}

Notes:

- Coeficientes estimados (Erros padrões entre colchetes)

- Representação P-valor: ${ }^{* * *} \mathrm{p}<0.01,{ }^{* *} \mathrm{p}<0.05,{ }^{*} \mathrm{p}<0.10$

A presença de codeshare no modelo se mostra com efeito negativo na demanda $(-0,0469)$, ou seja, essas rotas possuíam demanda reduzida no período. Durante o período de blackout period, houve uma queda na demanda $(-0,2425)$, o que era esperado se esse período for comparado com o período de crises financeiras. Essa análise confirma em parte o que foi dito na hipótese 5 - períodos de instabilidade financeira geram redução da demanda pelo transporte aéreo.

Como pode-se observar na Tabela 2, a presença da LCC Azul impactou positivamente a demanda, mostrando que a presença da Azul no mercado atraía mais passageiros, o que conforme visto em Hofer et al. (2008) era esperado. Em parte, essa análise corrobora com a hipótese 4 deste trabalho.

Com a lei da oferta e demanda, sabe-se que maiores preços possuem menores demandas, o modelo comprova isso através de yield uma vez que $1 \%$ de aumento nas tarifas aéreas gerou uma queda de $1,0036 \%$ no número de passageiros viajando. No entanto este valor pode estar inflado por não variar rota a rota, por isso interações desta variável com concorrência intermodal e com perfil da rota foram realizadas. Essas variáveis interagidas devem ser invariantes no tempo e variantes na rota.

Para a primeira interação concorrência intermodal é representada por intermod. Na segunda interação o perfil da rota é analisado através da variável business, a qual mostra a proporção de passageiros a negócios na rota. 
A interação da tarifa média tanto com a concorrência intermodal quanto com o perfil da rota torna possível uma análise das elasticidades-preço rota a rota. 0 efeito negativo no modelo da interação com intermod mostra que a possibilidade de fazer a rota de carro ou ônibus reduz a demanda de acordo com a tarifa, ou seja, quanto maior intermod, maior o módulo da elasticidade de yield. Já o efeito positivo com business mostra que a presença de passageiros a negócio reduz o módulo da elasticidade da tarifa média.

A partir das interações presentes no modelo foi possível calcular a elasticidade da variável yield conforme variação da rota, a qual é apresentada na Tabela 3.

Tabela 3: Elasticidade yield conforme variação do perfil da rota e da concorrência intermodal

\begin{tabular}{cccc}
\hline Intermod & $\mathbf{1 1 , 4 1 \%}$ & $\mathbf{3 5 \%}$ & $\mathbf{9 0 \%}$ \\
\hline In yield & $-1,0063$ & $-1,0119$ & $-1,0248$ \\
Business & $10 \%$ & $55,40 \%$ & $90 \%$ \\
In yield & $-1,0027$ & $-0,9984$ & $-0,9951$ \\
\hline
\end{tabular}

Ao avaliar a Tabela 3 é visto que em rotas nas quais $90 \%$ dos passageiros já realizaram por meio de outro modal, que não o aéreo, a demanda caiu em 1,0248\% frente a $1 \%$ de aumento nas tarifas. Enquanto para essa proporção em 11,41\% dos passageiros, valor médio, a redução da demanda foi de 1,0063\% frente ao mesmo $1 \%$ de aumento do preço. Isso mostra que diante da elevação das tarifas rotas com maior possibilidade de troca de modal leva a uma maior queda de demanda, isso porque além da desistência da viagem pode haver migração entre modais colaborando com essa redução.

Ainda na Tabela 3, ao analisar o perfil da rota, vê-se que quanto maior a proporção de passageiros corporativos menor é a queda na demanda. Quando essa proporção é de $90 \%$ a queda é de 0,9951\% para $1 \%$ de aumento em yield, já para $10 \%$ dos passageiros viajando a negócios a queda é de 1,0027\% perante os mesmos $1 \%$ de aumento. Isso devido a elasticidade do perfil do passageiro, turistas normalmente estão dispostos a alterarem seu destino ou até mesmo dias de viagem, enquanto uma pessoa a negócios possui dia, hora e local específico para determinadas ações, sendo eles menos elásticos ao aumento.

A interação yield com a dummy LCC é avaliada de forma diferente, uma vez que LCC é uma dummy apresentando apenas dois valores, 1 ou 0, ou seja, existe ou não existe. Diante disso a interação mostra que a elasticidade para mercados com a presença de empresas de baixo custo é menor do que para mercados sem essa presença. A existência da empresa Azul na rota representa uma queda na demanda de 0,4890\% para um aumento de $1 \%$ nas tarifas, enquanto para a mesma rota sem a concorrência dessa empresa a queda é de 1,0036\% para mesmo aumento. Ou seja, como empresas LCC ofertam com tarifas menores, os passageiros não desistem tanto da viagem devido à possibilidade de trocar de companhia aérea e continuar pagando um preço aceitável para os padrões pessoais. Essa análise afirma a hipótese 4.

A renda de uma pessoa ou família normalmente dita o consumo dos mesmos, com esse pensamento maiores rendas estão relacionadas a maiores consumidores, essa hipótese é válida para o modelo de transporte aéreo brasileiro uma vez que um aumento de $1 \%$ na renda está relacionado a um aumento de $0,1947 \%$ da demanda.

Percebe-se ainda pela regressão que um aumento na densidade populacional gera demandas maiores, ou seja, quanto mais povoada uma região, maior o número de pessoas viajando. Em outras palavras, um aumento de $1 \%$ na densidade populacional da rota gera um aumento de $1,2925 \%$ na demanda.

\subsection{Simulação}

A partir do modelo visto na Tabela 2 é possível estimar o impacto causado na demanda por um aumento dos custos das empresas. Como esse trabalho visa analisar o impacto de uma possível taxação ambiental sobre a demanda, um aumento de custos devido a essa taxa foi estimado.

Em 2012 a EU Emissions Trading System (EU-ETS) declarou que iria implementar licenças ambientais 
para empresas aéreas, inicialmente pensaram em uma taxa de 15 euros por tonelada de $\mathrm{CO}_{2}$ emitido, enquanto Iannone (2011) extrapolou esses valores e analisou taxas entre 10 e 30 euros.

No período do estudo em análise, segundo o Banco Central, a cotação média do euro foi de $\mathrm{R} \$ 2,75$, portanto a taxa aplicada por tonelada de $\mathrm{CO}_{2}$ de 15 euros é na verdade de $\mathrm{R} \$ 41,25$. A partir dessas taxações o aumento dos custos médio das viagens foi calculado, para assim ser possível analisar o impacto da taxação ambiental sobre a demanda. Essa análise foi possível utilizando a relação custo e preço dado por passthrough apresentado na seção anterior.

Para os dados analisados primeiramente foi utilizada a metodologia ICAO (2016) para quantificar as emissões de cada voo. Sabendo-se quantas toneladas de $\mathrm{CO}_{2}$ é emitido por rota é possível estimar o aumento dos custos das empresas, para as taxas de 10, 15 e 30 EUR o aumento nos custos foi de respectivamente $0,97 \%, 1,45 \%$ e $2,90 \%$. Com isso em mente diversos casos foram simulados para se analisar proporcionalmente a perda na demanda. Inicialmente foi realizada uma previsão do tráfego no cenário base analisado, além de uma previsão do tráfego no cenário em que a taxação é aplicada, a partir disso é possível ter a perda proporcional de demanda entre o modelo previsto e o simulado previsto.

A perda proporcional de demanda entre o previsto e o simulado previsto para o modelo completo mostra que uma taxa de 10 EUR gera um aumento de $0,97 \%$ nos custos, o que irá gerar uma queda de 5,50\% na demanda. Para a taxa de 15 EUR como o aumento dos custos é maior, a queda da demanda também é relativamente mais alta, 5,54\%, enquanto para 30 EUR de taxa a redução de demanda simulada é de 5,66\%. Com isso é visto que a queda não acontece de forma linear, até mesmo devido ao tipo de modelo e equação utilizada. Tudo isso constata o que foi afirmado na hipótese 1.

Diferentes características do mercado podem impactar de forma diferente a demanda. Levando isso em consideração foram realizadas algumas segmentações do mercado para o estudo da reação conforme essas características distintas, sendo elas respectivamente, existência de crise financeira, região destino e perfil da rota. A Figura 1 apresenta os resultados do impacto para cada uma dessas segmentações, e nela a largura das colunas está relacionada ao tamanho da amostra com a característica em análise.

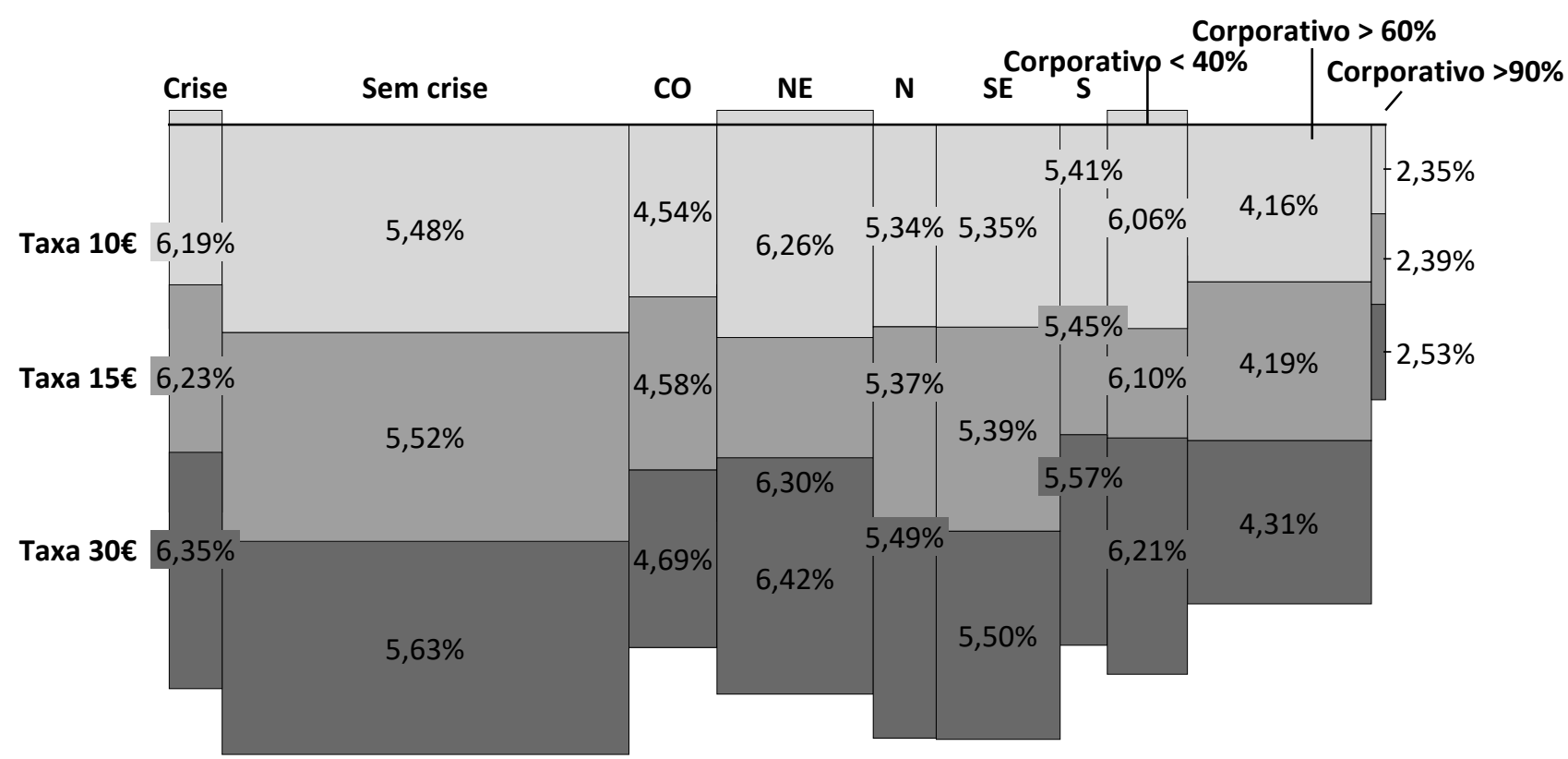

Figura 1. Redução da demanda devido à taxação ambiental segundo as segmentações de crise financeira, região destino de viagem e perfil da rota

\subsubsection{Crise financeira}

Examinando a presença do período de crise financeira global, 2008, comprova-se o que foi abordado anteriormente, que períodos de crise ocasionam maiores quedas de demanda proporcional do que em 
anos sem essa presença, Figura 1. Novamente temos evidências confirmatórias da hipótese 5, ou seja, a instabilidade econômica causa impacto na demanda

\subsubsection{Região destino}

A região destino da viagem pode ser um dos indicadores do motivo da viagem. Por exemplo, a região Nordeste brasileira é uma das regiões com maiores atrativos turísticos para os brasileiros devido às praias e ao clima do país, portanto é esperado uma queda proporcional maior na demanda se comparado a destinos mais corporativos. As regiões Sudeste, Sul e Norte podem atrair tanto passageiros turísticos quanto business, portanto uma queda intermediária é esperada. E o Centro-Oeste atende a capital política brasileira, o que torna essa região menos propensa à grande queda de demanda proporcional ao caso previsto versus previsto simulado.

A partir da Figura 1 é possível afirmar que a hipótese 3 é tida como verdadeira, isso porque essa hipótese diz que a região destino da viagem interfere na reação da demanda à aplicação da taxa. Pela análise realizada vê-se que o destino influencia no perfil do passageiro, o que também impacta no nível de reação da demanda.

\subsubsection{Perfil da rota}

0 perfil da rota é tratado por tipo de passageiro que viaja. Três tipos de perfis foram analisados, rotas com menos de $40 \%$ dos passageiros viajando a negócios, ou seja, rotas mais turísticas; rotas com mais de $60 \%$ de passageiros corporativos; e rotas com alta concentração corporativa, acima de $90 \%$. Pessoas viajando a turismo são mais flexíveis, voam muitas vezes de acordo com o preço e não de acordo com o local e dia, o que os tornam mais elásticos aos preços. A Figura 1 mostra exatamente isso, que quanto mais corporativa a rota é, menos a demanda é afetada ao aumento das tarifas.

Tabela 4: Resultados da estimativa 2SLS e LIML

\begin{tabular}{|c|c|c|c|}
\hline & (1) $2 \mathrm{SGMM}$ & (2) $2 S L S$ & (3) LIML \\
\hline \multirow[t]{2}{*}{ In grav pop density } & $1,2925^{* * *}$ & $1,2908 * * *$ & $1,2908 * * *$ \\
\hline & {$[0,079]$} & {$[0,079]$} & {$[0,079]$} \\
\hline \multirow[t]{2}{*}{ In grav income } & $0,1947 * * *$ & $0,1960 * * *$ & $0,1960 * * *$ \\
\hline & {$[0,026]$} & {$[0,026]$} & {$[0,026]$} \\
\hline \multirow[t]{2}{*}{ In yield } & $-1,0036^{* * *}$ & $-1,0012^{* * *}$ & $-1,0011 * * *$ \\
\hline & {$[0,166]$} & {$[0,166]$} & {$[0,166]$} \\
\hline \multirow[t]{2}{*}{ codeshare majors } & $-0,0469 * * *$ & $-0,0470 * * *$ & $-0,0470 * * *$ \\
\hline & {$[0,014]$} & {$[0,014]$} & {$[0,014]$} \\
\hline \multirow[t]{2}{*}{ blackout period } & $-0,2425^{* * *}$ & $-0,2422 * * *$ & $-0,2422 * * *$ \\
\hline & {$[0,016]$} & {$[0,016]$} & {$[0,016]$} \\
\hline \multirow[t]{2}{*}{ global fin crisis } & $-0,0216$ & $-0,0223$ & $-0,0222$ \\
\hline & {$[0,015]$} & {$[0,015]$} & {$[0,015]$} \\
\hline \multirow[t]{2}{*}{ LCC startup } & $1,9179 * * *$ & $1,9092 * * *$ & $1,9112 * * *$ \\
\hline & {$[0,353]$} & {$[0,357]$} & {$[0,358]$} \\
\hline \multirow[t]{2}{*}{ In yield $\mathrm{x}$ intermod } & $-0,0236 * * *$ & $-0,0233 * * *$ & $-0,0234 * * *$ \\
\hline & {$[0,008]$} & {$[0,008]$} & {$[0,008]$} \\
\hline \multirow[t]{2}{*}{ In yield $x$ business } & $0,0094 * * *$ & $0,0093 * * *$ & $0,0094 * * *$ \\
\hline & {$[0,003]$} & {$[0,003]$} & {$[0,003]$} \\
\hline \multirow[t]{2}{*}{ In yield $x$ LCC start } & $-0,5146 * * *$ & $-0,5121 * * *$ & $-0,5126 * * *$ \\
\hline & {$[0,097]$} & {$[0,099]$} & {$[0,099]$} \\
\hline Estatística RMSE & 0,3404 & 0,3399 & 0,3400 \\
\hline Estatística F & 1091,429 & 1088,530 & 1088,184 \\
\hline KP estatística & 137,6413 & 137,6413 & 137,6413 \\
\hline KP_P valor & 0,0000 & 0,0000 & 0,0000 \\
\hline J estatística & 0,3213 & 0,3213 & 0,3212 \\
\hline J_P valor & 0,8516 & 0,8516 & 0,8516 \\
\hline Estatística CD Weak & 41,8366 & 41,8366 & 41,8366 \\
\hline Estatística KP Weak & 24,3265 & 24,3265 & 24,3265 \\
\hline Número de observações & 25347 & 25347 & 25347 \\
\hline
\end{tabular}




\section{CHECK DE ROBUSTEZ E LIMITAÇÕES}

Os valores calculados para a elasticidade no modelo principal apresentado pela Equação 1 tiveram a validade e sensibilidade verificados pelo check de robustez. Foram verificados os resultados através da análise do modelo calculado por outros estimadores, os quais podem ser vistos na Tabela 4.

A Tabela 4 apresenta o resultado pelos estimadores 2SLS e LIML, e apresenta novamente o resultado do 2SGMM para melhor visualização das diferenças. De acordo com Angrist e Pischke (2008), se os resultados dos estimadores 2SLS e LIML forem similares então os instrumentos utilizados podem ser aceitos, isso porque, segundo os autores, LIML é menos preciso que 2SLS, porém é também menos viesado. Observa-se, no entanto, que o modelo LIML assume uma forte hipótese de distribuição independente e identicamente distribuído (IID), o que explica a escolha do modelo 2SGMM como estimador do trabalho.

Algumas limitações do trabalho a serem consideradas são a cotação aproximada do euro e a não variação da taxa ao longo dos anos. Além da falta de uma análise mais aprofundada da redução das emissões em números, afinal foi quantificada a redução da demanda, porém não a redução da oferta e do consumo de combustível, para então ser possível quantificar a queda das emissões dos gases CO2. Outra consideração existente, porém que não é bem tratada neste trabalho, é a aceitação dos passageiros à taxação ambiental.

\section{CONCLUSÕES}

Nos últimos anos o tema das emissões de gases do efeito estufa e seus efeitos potencialmente danosos sobre o planeta entrou definitivamente na pauta de governos ao redor do mundo, com destaque aos encontros internacionais realizados que visaram acordar entre os países formas de equacionar o problema. 0 mais recente dos encontros internacionais foi a COP22 realizado no ano de 2016, tal encontro, juntamente com a COP21 realizada em 2015, mostrou preocupação com aviação no nível de efeito estufa.

Na União Europeia essa preocupação das emissões do transporte aéreo vem sendo estudada há alguns anos, sendo que alguns países já analisaram até mesmo valores para uma taxação ambiental. Esse valor varia conforme o país, porém têm sido aplicados valores aproximados a 15 EUR desde 2010.

Como a taxação é aplicada por tonelada de $\mathrm{CO}_{2}$ emitido, um cálculo com base na calculadora ICAO (2016) foi realizado, quantificando assim as emissões de cada voo analisado. Com base nesse valor foi possível aplicar a taxa ambiental para análise do aumento dos custos e redução da demanda.

As principais contribuições do trabalho se deram nas análises da reação da demanda ao aumento dos custos por características variáveis do mercado. Como era esperada, a modelagem econométrica mostrou que a demanda reage com queda para aumento nas tarifas, porém mostrou também que diferentes características afetam na forma com que essa demanda cai. Por exemplo, diferentes regiões destino de viagem possuem diferentes reações, mostrando que regiões mais turísticas sofrem maior queda de demanda do que regiões mais relacionadas a negócios, isso para um mesmo aumento nos custos. Ou seja, o tipo de viagem e perfil de passageiros também afetam diretamente nessa reação.

O estudo pode ser considerado de extrema importância ao avaliar a redução da demanda a choques no custo, pois isso impacta não somente o setor aéreo, mas também impacta diversos setores como o comércio em regiões turísticas e a redução de negócios de empresas, o que pode acarretar uma crise econômica nas companhias ou até mesmo no país. Portanto, esse tipo de estudo é necessário antes da real aplicação de um taxa ambiental, para não haver mais perdas do que ganhos em todo o mercado.

\section{AGRADECIMENTOS}

Os autores agradecem à Capes, CNPq (auxílio 2013/14914-4) e Fapesp (auxilio 15-1944-1) pelo auxílio financeiro.

\section{REFERÊNCIAS}

Andersen, M. S. (2010). Europe's experience with carbon-energy taxation. Sapiens, v. 3, n. 2, p. 1-11. Disponivel em: <https://sapiens.revues.org/1072>.

Angrist, J. D. e J. S. Pischke (2008). Mostly harmless econometrics. United Kingdom: Princeton University Press. 
Betiolo, C. R.; G. C. Rocha e P. R. de C. Machado (2009). Iniciativas da aviação para a redução de emissões de CO². SIMPÓSIO DE TRANSPORTE AÉREO, 8., 2009. Anais... São Paulo, p. 401-409.

Bosquet, B. (2000). Environmental tax reform: does it work? a survey of the empirical evidence. Ecological Economics, v. 34, p. 19-32. DOI: 10.1016/S0921-8009(00)00173-7

Brouwer, R.; L. Brander e P. Van Beukering (2008). A convenient trut: air travel passengers willingness to pay to offset their CO2 emissions. Climate Change, v. 90, p. 299-313. DOI: 10.1007/s10584-008-9414-0

EUROSTAT (2013). Manuals and guidelines: environmental taxes: a statistical guide. Luxemburg: European Union, DOI:10.2785/47492

Grampella, M.; G. Martini; D. Scotti e G. Zambon (2016). The factors affecting pollution and noise environmental costs of the current aircraft fleet: an econometric analysis. Transportation Research Part A, v. 92, p. 310-325. DOI: 10.1016/j.tra.2016.06.013

Greene, W. H. (2007). Econometric analysis. 6. d. New Jersey: Pearson.

Hausman, J. A. (1996). Valuation of new goods under perfect and imperfect competition. In: Bresnahan, T. F. e R. J. Gordon (eds.) The economics of new goods. Chicago, IL, USA: University of Chicago Press. DOI: 10.3386/w4970

Hofer, C.; M. E. Dresner e R. J. Windle (2010). The environmental effects of airline carbon emissions taxation in the US. Transportation Research Part D, v. 15, p. 37-45. DOI: 10.1016/j.trd.2009.07.001

Hofer, C.; R. J. Windle e M. E. Dresner (2008). Price premiums and low cost carrier competition. Transportation Research Part E, v. 44, p. 864-882. DOI: $10.1016 /$ j.tre.2007.03.004

Iannone, K. (2011). Green Skies: effects of environmental taxation on the u.s. domestic airline industry. 2011. 48 p. Trabalho de conclusão de curso (Graduação) - Boston College, Boston, MA, USA. Disponível em: <http://hdl.handle.net/2345/2003>.

ICAO (2016). Carbon emissions calculator methodology. 2016, v. 9, 37p.

Jou, R. e T. Chen (2015). Willingness to pay of air passengers for carbon-offset. Sustainability, v. 7, p. 3071-3085. DOI: $10.3390 /$ su703307

Motta, M. (2004). Market definition, and the assessment of market power. In: United Kingdom: Cambridge University Press,

Oliveira, A.V. M. de; N. S. Ferreira e L. H. Salgado (2011). Liberalização econômica do transporte aéreo no Brasil: um estudo empírico dos dez primeiros anos. Transportes, v. 19, n. 3, p. 62-74. DOI: 10.14295/transportes.v19i3.369

Pavão, H. G. (1997). 0 gás carbônico atmosférico: variações e influência das queimadas em várias regiões do Brasil. Revista Brasileira de Geofísica, v. 15, n. 3, p. 311. D0I: 10.1590/S0102-261X1997000300011

Silva, R. W. da C. e B. L. Paula (2009). Causa do aquecimento global: antropogênica versus natural. Terra e Didática, v. 5, n. 1, p. 42-49. Disponível em: <https://www.ige.unicamp.br/terraedidatica/>.

Simões, A. F. e R. Schaeffer (2005). The brazilian air transportation sector in the context of global climate change: co2 emissions and mitigation alternatives. Energy Conversion and Management, v. 46, p. 501-513. DOI:

10.1016/j.enconman.2004.06.017

Wilhelm, D. (2015). Optimal bandwidth selection for robust generalized method of moments estimation. Econometric Theory, v. 31, n. 5, p. 1054-1077. DOI: 10.1017/S026646661400067X

Williams, R. C. (2002). Environmental tax interactions when pollution affects health or productivity. Journal of Environmental Economics and Management, v. 44, p. 261-270. DOI: 10.1006/jeem.2001.1237

Wooldridge, J. M. (2013). Introductory econometrics: a modern approach. 5. ed. Mason, OH: South-Western. 